\title{
Sessile axisymmetric drops in microgravity conditions
}

\author{
Amelia Carolina Sparavigna \\ Department of Applied Science and Technology, Politecnico di Torino, Torino, Italy
}

Email address:

amelia.sparavigna@polito.it

\section{To cite this article:}

Amelia Carolina Sparavigna, Sessile Axisymmetric Drops in Microgravity Conditions. American Journal of Modern Physics, Vol. 2, No. 5, 2013, pp. 251-254. doi: 10.11648/j.ajmp.20130205.13

\begin{abstract}
Fluid interfaces are producing several static and dynamic phenomena. These interfaces are governing, for instance, the shape of sessile droplets and the spread of liquids on surfaces. In this paper, we will discuss the shape of sessile axisymmetric drops on a solid surface and how it is depending on gravity, obtaining results that are in agreement with experimental observations under conditions of microgravity.
\end{abstract}

Keywords: Surface Tension, Wetting, Sessile Drops, ADSA, Microgravity

\section{Introduction}

Many static and dynamic phenomena can be observed when we have a system containing some fluid interfaces. We can see for instance, that the sessile drops tend to have a lenticular shape and that the water spreads on some surfaces whereas it is remaining in isolated drops on others. These phenomena can be understood through the concept of surface tension. From a molecular point of view, we can imagine a molecule and the forces acting on it: if a molecule is at the surface of a liquid, the net force on it is different, compared with that acting on a particle in the bulk. In fact, the net force acting on a molecule in the bulk is null in average, because this molecule is pulled equally by the close molecules of the liquid phase. A molecule at the surface of the fluid, however, feels an unbalanced force pulling it inwards, due to the lack of nearby molecules on the side of the vapour phase.

In order to study the surface tension and interfacial forces between liquids and solid substrates, the analysis of the shape of sessile droplets is one of the most used methods. There are several reasons supporting the preference for this method, in particular it is easy to apply and requires small quantities of liquid. [1] Currently, the method is combined with the use of image processing and numerical computations on personal computers to fit the recorded data. When applied to axisymmetric droplets, the overall procedure is defines as ADSA, Axisymmetric Drop Shape Analysis [2,3]. The calculation of the form of the droplets is based on numerical solutions of Young-Laplace equation.

Here we will discuss the shape of axisymmetric droplets, according to the ADSA method. We will see in particular its dependence on the gravity, comparing it with the experimental observations obtained under the conditions of microgravity.

\section{Microgravity and Interfacial Phenomena}

Actually, surfaces and interfaces need more investigation. For instance, a better understanding of the interfacial phenomena, such as the wetting, will help improving materials processing [4]. Moreover, interfaces dominate the properties and behaviour of advanced composite materials [5]. Since the control of wetting and spreading is still posing scientific and technological challenges, it deserves some studies in the regime of microgravity. This peculiar environment provides scientists with an excellent opportunity to investigate all those features of wetting and surface tensions normally masked by the Earth's gravity [4].

The term microgravity, indicated by the symbol $\mu \mathrm{g}$, is a synonym of weightlessness, and corresponds to an environmental condition where the gravitational force is very small. Then, the best laboratory to investigate microgravity is the International Space Station [6]. Another possibility is to use reduced gravity aircrafts, that is, special airplanes in parabolic flights, or the microgravity drop towers. These are structures used to produce a controlled period of weightlessness. Air bags and magnetic or mechanical brakes are arresting the fall of the experimental load. The period of weightlessness is of a few seconds, 
when the sample is freely falling in the tube. During its flight, the sample is characterized by means of the instruments included in the experimental load. In fact, the first drop tower was the Leaning Tower of Pisa, which is popularly supposed to have been used by Galileo to demonstrate that the bodies with different masses fall with the same acceleration [7].

The research of interfacial phenomena in microgravity focuses on how an interface acquires and maintains its shape [8-10]. For instance, we can see that in the condition of a reduced gravity, droplets assume a spherical shape (see the Figure 1).
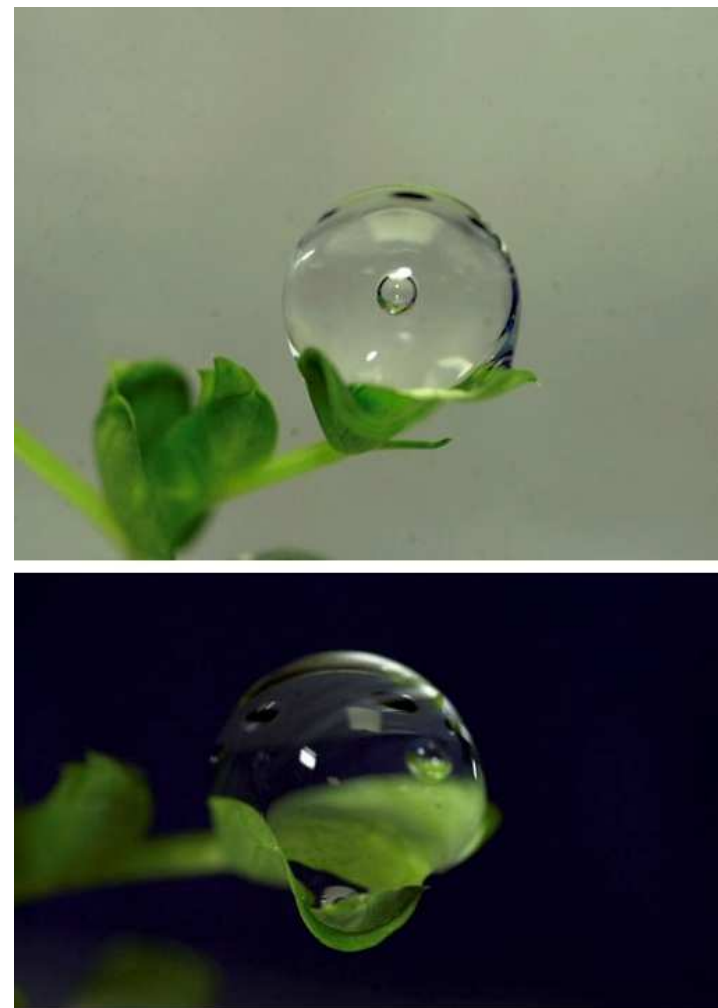

Figure 1. Droplets and bubbles in the space garden of the International Space Station (Courtesy: NASA).

Besides the analysis of static surfaces, the dynamics is also studied in response to heating or cooling, and to chemical influences. On the ground, the gravity causes buoyancy, which is generating convection. In the space, buoyancy disappears; however, a different type of convection exists, originated by the surface tension, which is depending on the temperature and, in the case of a mixture, on its concentration. Accordingly, if a temperature or concentration distribution exists, a distribution of the surface tension appears, with some flow occurring towards the places where the surface tension is higher. This mechanism is the Marangoni convection [11]. It is therefore important to study the surface tension in microgravity conditions, because it has a great impact in the micro-world, where the gravity becomes less significant due to the small scale of the environment, and because this study can help improving the micro-fluid technology on the ground [11].

\section{Contact Angle and Wetting}

When a drop of liquid is placed on a solid surface, a triple linear interface is formed where the solid, liquid and vapour phases coexist. This triple line will move in response to the forces arising from the three interfacial tensions until an equilibrium position is established. In Figure 2 we can see a model of a drop of liquid (L) on a solid surface (S) with vapour $(\mathrm{V})$ as the third phase. The angle $\theta$ between the solid surface and the tangent to the liquid surface at the line of contact with the solid is the contact angle. At equilibrium, the tensions $\gamma$ will be in balance and thus we have the condition: $\gamma_{\mathrm{VS}}=\gamma_{\mathrm{LS}}+\gamma_{\mathrm{VL}} \cos \theta$. The origin of the contact angle is therefore in the cohesive forces between the molecules in the liquid drop and the adhesive forces between the molecules and the solid surface.



Figure 2. A molecule at the surface of a drop is subjected to a net force, which is the origin of the surface tension. On the right of the drop, we can see the contact angle $\theta$.

We can see then that some surfaces have a very high contact angle for water, while on other surfaces it is so low that we cannot measure it [12]. A surface having a good affinity for water has a low contact angle. Such a surface is hydrophilic. If a surface has preponderantly non-polar groups on it, such as a polymeric surface, the surface is hydrophobic and the contact angle will be large. Wetting is determined by the equilibrium contact angle too. If $\theta<90^{\circ}$, the liquid is said to wet the solid; if $\theta=0$, there is a complete or perfect wetting; if $\theta>90^{\circ}$, the liquid does not wet the solid [13]. However, these features of a surface can be controlled by means of some specific treatments, such as those using the atmospheric plasma $[14,15]$.

The measurement of contact angle is therefore a simple method to gain qualitative information about the surface. Many surfaces display an apparent hysteresis, giving different values of the contact angle when the measurement is obtained from a drop of increasing size (advancing contact angle) or of diminishing size (receding contact angle). A cause of this hysteresis is in the roughness of the surface, and then the difference between advancing and receding contact angles is used to obtain information about the nature of the surface [14]. 


\section{The Young-Laplace Equation and its Solution}

ADSA methods are based on the best fit between the shape of the drops, as experimentally evidenced, and the numerical solution of the mathematical model given by the Laplace-Young equation for capillarity [2].

The general form of the Laplace-Young equation describes the capillary pressure difference sustained across an interface, due to the surface tension. In particular, the equation relates the pressure difference $\Delta p$ to the shape of the surface:

$$
\Delta p=\gamma\left(\frac{1}{R_{1}}+\frac{1}{R_{2}}\right)
$$

where $R_{1}, R_{2}$ are the principal radii of curvature and $\gamma$ the surface tension. Therefore, the variation of the pressure is given by two contributions: the change in hydrostatic pressure and the change due to the curvature of the drop. However, the sessile drops do not possess a constant curvature, and then Equation 1 needs a local coordinate system. A spherical coordinate system is suitable to describe the local curvature. Figure 3 illustrates such a system with its origin located at the apex of the drop [1-3].

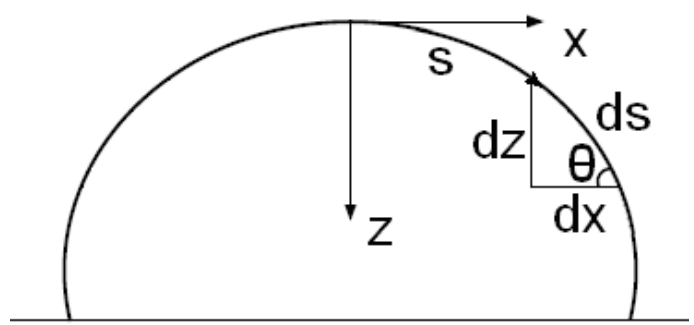

Figure 3. Spherical coordinate system used to describe the local curvature of the sessile drop. Its origin is at the apex of the drop.

The model and the numerical method used to determine the shape of the sessile drops are given in Ref.2. Equations are:

$$
\begin{aligned}
& \frac{d x}{d s}=\cos \theta \\
& \frac{d z}{d s}=\sin \theta \\
& \frac{d \theta}{d s}=2 b+c z-\frac{\sin \theta}{x}
\end{aligned}
$$

In Equation 2, $s$ is the arc-length, $b$ the curvature at the origin of the coordinates and $c$ the capillarity constant of the system, $g \Delta \rho / \gamma$, where $g$ is acceleration of gravity. To apply the model (2) to investigate the shape of droplets in microgravity, we can use the Runge-Kutta method of solution in a Fortran program. The capillarity constant in the regime on normal gravity, is assumed to have the value of $13.5 \mathrm{~cm}^{-2}$, according to Reference 2 . To compare the effect of microgravity (here a value of $10^{-6} \mathrm{~g}$ is used), let us consider the drops having a fixed volume $\left(1 \mathrm{~cm}^{3}\right)$, for several apex curvatures.

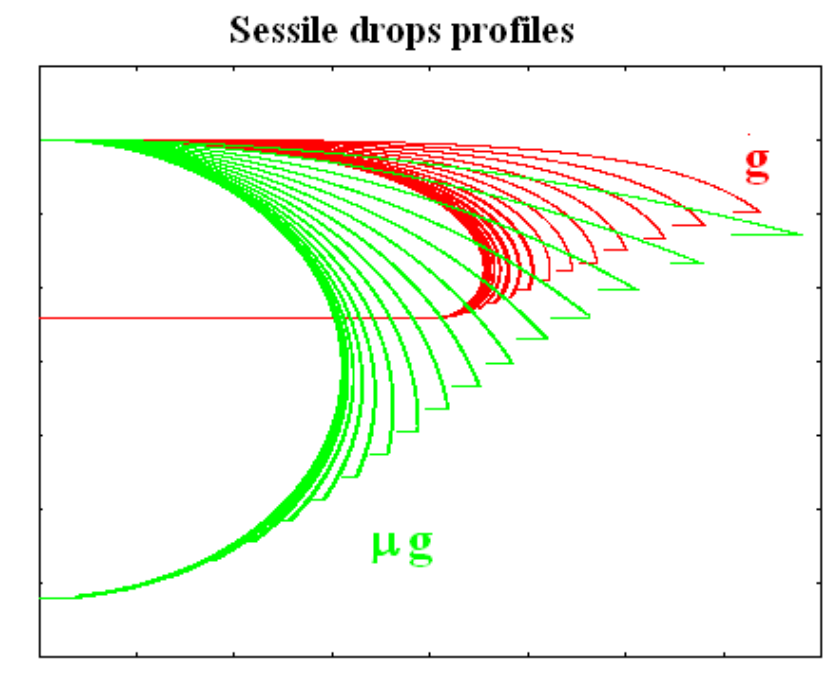

Figure 4. Profiles of sessile drops, which have the same volume but different apex curvatures, in the case of gravity (red curves) and microgravity (green curves) conditions.

The profiles of droplets in the coordinate system of Figure 3 are given in the Figure 4 . We have, in red, the profiles of droplets in the normal gravity field, and, in green, those in microgravity. The droplets have the same fixed volume. We can see several droplets having different contact angles with the horizontal surface, represented by a short horizontal line segment (the droplets are displayed as having the same apex). We can see that the shape in normal gravity is more flat than that of a droplet, which has the same volume and the same contact angle, but in the condition of microgravity. In microgravity, the shape of droplets can be even spherical, and this is what is observed in experiments [8].

\section{Conclusions}

Reference 8, published in 2012, is presenting a study aimed to the development of a database of the contact angles of sessile drops in reduced gravity. According to the authors, such a database does not exist, but the creation of it is fundamental for investigating the role of heat and mass transfer in reduced gravity and future engineering designs. In fact, this is in agreement with Reference 11, which is remarking the importance of studies in microgravity, the results of which can be applied to micro- and mesoscopic systems where the role of gravity is reduced.

In Reference 8, the authors investigated water and ethanol on PTFE and aluminium surfaces. They observed that in the reduced gravity environment, the droplet shape is described adequately by the Young-Laplace equation. A variation is occurring in the diameter and height upon the surface, towards a sphere-like volume. The dimensional analysis of the Bond number $B$ and capillary length, $\left(B=\left(r / L_{\mathrm{c}}\right)^{2}=g \Delta \rho\right.$ $r^{2} / \gamma$, where $r$ is the wetting radius and $L_{\mathrm{c}}$ is the capillary 
length), correlated well with the change in the wetted diameter and height of the droplet. In fact, the triple line perimeter of the drops is lower in reduced gravity than in normal gravity condition.

Then, according to [8], the Young- Laplace equation can be used to determine the contact angle in reduced gravity for small droplets, but it is not optimal to describe the contact angle for larger drops. Moreover, hysteresis exists because of drop pinning due to the roughness of the surface, and the pinning can be affected by gravity too. Therefore, the Reference 8 is claiming that more experiments are necessary to understand the behaviour of large droplets and to improve the related theory.

In this paper, we have proposed a short discussion of the problem that is suitable for teaching purposes, in particular to show an application of the Young-Laplace equation, application which is involving image processing and numerical methods, besides being important for industrial problems. The aim is that of stimulating the students towards the study of the problems connected with a microgravity environment.

\section{References}

[1] M. Hoorfar, and A. Wilhel Neumann, "Axisymmetric Drop Shape Analysis (ADSA)," in Applied Surface Thermodynamics, Second Edition, Yi Zuo Editor, CRC Press, 2010, pp.107-174.

[2] O.I. del Río, and A. W. Neumann, "Axisymmetric Drop Shape Analysis: Computational Methods for the Measurement of Interfacial Properties from the Shape and Dimensions of Pendant and Sessile Drops," Journal of Colloid and Interface Science, vol. 196, pp. 136-147, 1997.

[3] M. Hoorfar, Development of a PC version for axisymmetric drop shape analysis (ADSA), Thesis, Department of Mechanical and Industrial Engineering University of Toronto. 2001.

[4] M.J.B. Rogers, G.L. Vogt, and M.J. Wargo, "Microgravity Science Primer, A Teacher's Guide with Activities in Science, Mathematics, and Technology," EG-1997-08-110-HQ, NASA, 1997, at the web page http://www.nasa.gov/
[5] M. Lappa, Fluids, Materials And Microgravity: Numerical Techniques And Insights Into Physics, Elsevier, 2004.

[6] Unlocking Mysteries in Microgravity, NASA Facts, National Aeronautics and Space Administration, Glenn Research Center, FS-1999-07-007-GRC, published at the site http://www.nasa.gov/centers/glenn/about/fs07grc.html

[7] C.G. Adler, and B.L. Coulter, "Galileo and the Tower of Pisa experiment," American Journal of Physics, vol. 46(3), pp. 199-201, March 1978.

[8] A. Diana, M. Castillo, D. Brutin, and T. Steinberg, "Sessile Drop Wettability in Normal and Reduced Gravity," Microgravity Sci. Technol., vol. 24, pp. 195-202, Springer, 2012, DOI 10.1007/s12217-011-9295-0

[9] Zhi-Qiang Zhu, D. Brutin, Qiu-Sheng Liu, Yang Wang, A. Mourembles, Jing-Chang Xie, and L. Tadrist, "Experimental Investigation of Pendant and Sessile Drops in Microgravity," Microgravity Sci. Technol., vol. 22. pp.339-345, 2010, DOI 10.1007/s12217-010-9224-7

[10] D. Brutin, Zhi-Quiang Zhu, O. Rahli,Jing-Chang Xie, Qui-Sheng Liu, and L. Tadrist, "Sessile Drop in Microgravity: Creation, Contact Angle and Interface," Microgravity Sci. Technol, vol. 21, pp.s67-s76, Springer, 2009, DOI $10.1007 / \mathrm{s} 12217-009-9132-\mathrm{x}$

[11] H. Kawamura, "Fluid Science under Microgravity," JAXA, Japan Aerospace Exploration Agency, at http://www.jaxa.jp/article/special/kibo/kawamura_e.html

[12] S.W. Rienstra, "The Shape of a Sessile Drop for Small and Large Surface Tension," Journal of Engineering Mathematics, vol. 24, pp.193-202, 1990

[13] D. L. Schodek, P. Ferreira, and M. F. Ashby, Nanomaterials, Nanotechnologies and Design: An Introduction for Engineers and Architect, Butterworth-Heinemann, 24/mar/2009, Pag.409.

[14] R. Wolf, A.C. Sparavigna, "Role of Plasma Surface Treatments on Wetting and Adhesion," Engineering, vol. 2(6), pp.397-402, 2010, DOI: 10.4236/eng.2010.26052

[15] A.C. Sparavigna, "Plasma Treatment Advantages for Textiles," arXiv, 2008, arXiv:0801.3727 [physics.pop-ph]

[16] Russell Stacy, Contact Angle Measurement Technique for Rough Surfaces, Thesis, Michigan Technological University, 2009. 